\title{
LES MONNAIES DE LA RÉPUBLIQUE. UN RETOUR SUR LES IDÉES MONÉTAIRES DE JEAN BODIN
}

\author{
Jérôme Blanc * \\ Version révisée (février 2005) pour les \\ Cahiers d'économie politique
}

\section{Résumé}

Les écrits monétaires de Bodin portent principalement sur le rapport des monnaies à la souveraineté. Bodin cherche à concevoir un système de monnaies à l'épreuve des contrefaçons, altérations et dégradations. Après avoir exposé sa conception de la monnaie et de la fausse monnaie, on expose la façon dont Bodin cherche à contraindre l'exercice de la souveraineté monétaire : en soumettant la manipulation des monnaies non à la loi mais au contrat, en construisant un système de monnaies idéal et en définitive en définissant une contrainte internationale. La souveraineté monétaire, absolue dans son principe, apparaît ainsi contrainte dans son exercice.

\begin{abstract}
Bodin's monetary writings are mainly centred on the relationship between the currencies and sovereignty. He aims at designing a monetary system that would be forgery, debasement and degradation proof. Having explained his conception of money and false money, we expose the way Bodin seeks to constrain the exercise of monetary sovereignty : by submitting debasements not to the law but to the contract, by setting up an ideal monetary system and finally by defining an international constraint. The monetary sovereign power, whose principle is absolute, appears to be under constraint in its exercise.
\end{abstract}

Classification JEL : B11, B31, E42

\footnotetext{
* Maître de conférences à l'Université Lumière Lyon 2. Rattaché au Centre Auguste et Léon Walras / LEFI. Institut des sciences de l'homme (ISH), 14, avenue Berthelot, 69007 Lyon, Tél : 04727264 07, Fax : 04727265 55. Adresse électronique : jerome.blanc@,univ-lyon2.fr
} 


\section{LES MONNAIES DE LA RÉPUBLIQUE. UN RETOUR SUR L'ANALYSE MONÉTAIRE DE JEAN BODIN}

\section{Introduction}

Jean Bodin est surtout reconnu en économie pour avoir jeté les bases d'une relation entre l'offre de monnaie et le niveau des prix, lors de la célèbre controverse née de la publication des Paradoxes de Malestroit en $1566^{1}$. Son analyse de la monnaie ne se limite cependant pas à cette question qui a pris une importance démesurée au point d'effacer le reste de ses réflexions économiques ${ }^{2}$. Pour Bodin, la monnaie relève en effet de la problématique de la souveraineté, qui constitue la pierre angulaire de son oeuvre. A ce titre, elle retient l'attention d'un chapitre entier des Six livres de la République (le chapitre 3 du sixième livre), chapitre repris dans sa totalité lorsque Bodin révise sa Réponse aux Paradoxes de Malestroit. L'analyse monétaire de Bodin constitue donc, à part entière, un élément de l'élaboration théorique de la puissance souveraine que représentent les Six livres de la République.

Les idées présentes dans la Réponse (1568), la République (1576), et le Discours (1578)³ ne prennent pourtant ni la forme, ni la profondeur d'un traité des monnaies. Le domaine de

1. Je remercie Jean-Michel Servet, Jean-Pierre Potier et Estrella Trincado qui, à diverses occasions, m'ont permis d'améliorer ce texte, de même qu'un référé anonyme de la revue pour sa lecture précise et efficace. Ainsi que le veut la formule, je reste néanmoins seul responsable des erreurs qui pourraient subsister.

2. Schumpeter (1983, I, pp. 432-438) donne une vision d'ensemble et critique des origines de la théorie quantitative, même s'il omet de parler de Nicolas Copernic (Voir pour cela Guggenheim 1978, pp. 21-29).

3. Les textes de Bodin auxquels nous ferons référence sont La méthode de l'bistoire (1566), La Response de Maistre Jean Bodin advocat en la cour au Paradoxe de Monsieur de Malestroit, touchant l'encherissement de toutes choses, et le moyen d'y remedier (1568), Les six livres de la République (1576, édition de 1583 publiée en abrégé par Gérard Mairet en 1993 et édition de 1593 publiée chez Fayard en 1986), le récit que fait Bodin des Etats généraux de 1576-77 (Recueil de tout ce qui s'est négocié en la compagnie du tiers Estat de France..., 1577), le Discours de Jean Bodin, sur le rebaussement et diminution tant d'or que d'argent, et le moyen d'y remédier, aux Paradoxes du Sieur de Malestroit (1578), et l'Exposé du droit universel (1580).

On trouve désormais sur le site Gallica de la Bibliothèque nationale de France des éditions numérisées des Paradoxes de Malestroit, de la Response de Bodin, de son Discours, des Six livres de la République et d'autres textes encore. En 1997 est parue une édition traduite en anglais de la Réponse de Bodin mentionnant les différences entre les éditions de 1568 et 1578 et reproduisant les Paradoxes de Malestroit (Response to the Paradoxes of Malestroit, Translated by $\mathrm{H}$. Tudor and R. W. Dyson, Bristol : Thoemmes, 1997). Elle a donné lieu à quelques articles prenant le parti de réévaluer le quantitativisme de Bodin — mais avec des résultats opposés : particulièrement 
prédilection de Bodin est en effet le droit, pas la monnaie, et ses considérations dans ce domaine n'ont pas de fin théorique mais bien pratique. Ces considérations sont celles d'un analyste à l'intelligence perçante qui parvient à mettre en forme de façon cohérente la masse d'informations qui lui parvient, et qui, par ailleurs, évite certains écueils présents dans l'ensemble de son oeuvre puisque, hormis au sujet de l'usure, on ne peut trouver de trace dans son analyse monétaire de l'obscurantisme dont il fait parfois preuve ${ }^{4}$. Dans ce cadre, dépendant du contenu théorique de la République et de l'environnement historique de l'auteur, l'analyse monétaire de Bodin consiste en une série de conseils de bon gouvernement des monnaies. Or ces conseils ne portent pas sur la question de l'abondance des métaux précieux, à laquelle il ne donne ni même ne cherche de solution. Ce qui intéresse le plus Bodin en matière monétaire est en effet la question de la fausse monnaie. La raison en est simple, cohérente, logique : contrairement au problème de l'abondance des métaux précieux, celui de la fausse monnaie met en cause ce qui constitue l'élément clé d'une bonne partie de son œuvre, c'est-à-dire la souveraineté.

Ces conseils doivent guider l'élaboration d'un nouveau système de monnaies, dont la finalité est d'annihiler la possibilité même du faux-monnayage. Ce système idéal est le résultat de forts prémices, d'une logique des idées parfois implicite dont la mise en lumière constitue l'objet de ce texte.

Le coeur du discours de Bodin se situe dans la problématique, courante à l'époque, de la fausse monnaie, et dont il faut lever les ambiguités pour mieux saisir la conception implicite de la monnaie qu'elle suppose (I). L'objectif de Bodin est d'annihiler le faux-monnayage. Il s'engage

O’Brien (2000), préfacier de l'édition anglaise, et Arestis et Howells (2002). Nous traitons de la question dans Blanc (2005).

4. Bodin condamne en effet sans appel le prêt à intérêt, même du plus faible taux, avec une verve mystique : "L'usure, que les Hébreux appellent morsure, non seulement ronge le debteur jusques aux os, mais aussi succe tout le sang et la mouëlle des os » (Bodin 1593, V/2, p. 60). L'obscurantisme dont nous parlons - ou du moins ce qui apparaît aujourd'hui comme tel - est surtout présent hors de son oeuvre économique. Goyard-Fabre (1989, pp. 9-10) parle d'ambiguïté : "il pense la République - la Res publica - déjà en moderne, mais encore en ancien. Le contenu de ses ouvrages, répétitif à soubait, expose un droit des républiques tourné vers la modernité politique, mais qui se dessine sur des horizons médiévaux chargés d'un mysticisme où l'on a cru voir s'entremêler parfois de la superstition ». Voir ainsi ses considérations sur les proportions harmoniques, géométriques et arithmétiques auxquelles il prête des qualités de mécanisme caché des républiques, sa curieuse condamnation du théâtre (Bodin 1593, VI/1, pp. 24-25), mais aussi, au-delà de la République, sa Démonomanie des sorciers, publiée pour la première fois en 1580 et ayant connu un immense succès, etc. Baudrillart (1853) résume ainsi l'ambiguité : "A moitié plongé dans le Moyen âge par sa foi superstitieuse à la magie, il s'avance jusqu'au XVIIIe siècle tant par ses vues hardies et fermes en matière politique que par sa claire compréhension des phénomènes économiques et monétaires. Aucun écrivain ne paraît avoir mieux marqué la limite des deux âges avec ce trait particulier qu'en lui ils se juxtaposent sans se confondre et coexistent sans se combattre ». 
sur cette voie en formalisant le domaine monétaire avec les notions de loi et de contrat, et tente ainsi de montrer la nécessité pour le souverain d'être juste en la matière (II). C'est de cette justice qu'il fait dépendre la pérennité de la foi publique, elle-même condition de pérennité de la république. Mais en dernier recours, ne pouvant trouver à l'intérieur de sa construction théorique de la république les ressources contraignant le souverain à la vertu, Bodin est conduit à trouver cette contrainte dans le pouvoir des autres souverains (III). Au-delà de la question monétaire, il ressort une idée puissante qui relativise l'absolutisme de principe dont on caractérise souvent Bodin : celui-ci cherche, sans cesse, à limiter très concrètement l'exercice du pouvoir du souverain en l'enracinant dans le quotidien.

\section{De la monnaie au métal, ou la recherche d'une vraie et bonne monnaie}

L'analyse du discours monétaire de Bodin commence par la mise au clair de sa conception de la monnaie (A). Cette conception conduit à aborder le problème de la fausse monnaie (B), dont la racine doit être trouvée dans la falsification des métaux et non de la marque royale, dans le cadre d'une conception monétaire métalliste (C).

\section{A. Qu'est-ce que la monnaie?}

A aucun moment de son oeuvre économique, Bodin ne définit explicitement ce qu'il entend par monnaie. Il n'y a d'ailleurs pas de concept de monnaie à proprement parler dans ses écrits, mais une conception de la monnaie qui s'enracine dans un ensemble de réflexions sur les monnaies. C'est ainsi que, comme ses contemporains, Bodin parle en général des monnaies au pluriel, faisant des pièces la monnaie par excellence ou le fondement de la réflexion monétaire.

La monnaie apparaît de la sorte avant tout comme un moyen de paiement :

« la monnoye est une chose sainte qui ne doibt estre alteree, depuis qu’on luy a donné son vray titre et juste valeur» (Bodin 1568, p. 144).

Le titre est le terme approprié pour désigner la teneur en métal fin, or ou argent, de la pièce ; la valeur fait référence au cours légal que le «on », c'est-à-dire, sans le nommer, le prince, confère à cette pièce. Cette valeur s'exprime en une unité que Bodin ne définit pas.

Par ailleurs, la monnaie est ce qui «donne estimation et pris aux choses» (Bodin 1568, p. 84) ; c'est elle qui «doit reigler le prix de toutes choses» (Bodin 1593, VI/3, p. 117). Ceci renvoie à un usage de la monnaie comme unité de compte, car elle est cet instrument qui met en ordre et permet d'évaluer et fixer les prix des choses (le mot régler ne doit pas être assimilé au règlement d'une dette mais à un ordonnancement). A ce titre, la manipulation du cours par le souverain pose problème, «car si la monnoye (...) est muable et incertaine, il n'y a personne qui puysse faire estat au vray de ce qu'il a» (Bodin 1593, VI/3, p. 117). Il reste que la monnaie relève bien de la 
souveraineté, à l'instar des poids et mesures (Bodin 1593, I/10, p. 334). Il y a un autre problème : l'existence de plusieurs "livres de monnoye », c'est-à-dire plusieurs définitions (poids métallique) de la monnaie dans le royaume, au-delà de la définition tournois, complique la situation pour le peuple qui en général ne maitrise pas le change ${ }^{5}$.

Il faut souligner que Bodin ne développe à aucun moment une vision fonctionnelle de la monnaie. Il identifie des pratiques, portant sur des pièces et sur des unités de compte, mais ne développe pas de réflexion abstraite sur cette base. La monnaie est pour lui la généralité des monnaies et non un concept opératoire. Ainsi, si Bodin montre des capacités d'analyse des phénomènes monétaires par sa connaissance à la fois de la réalité présente et passée et des considérations savantes de son temps sur les monnaies, il ne développe pas de raisonnement monétaire abstrait. Par ailleurs, il réfléchit assez peu aux mécanismes du change. Il réduit la question monétaire au problème du métal et à son rapport à la souveraineté, se centrant sur le problème polymorphe de la falsification.

\section{B. Qu'est-ce que la fausse monnaie?}

Dans le système que développe Bodin, la monnaie se résume donc aux pièces métalliques. Or Bodin affirme qu'elles sont à la fois incommodes et impropres à conserver la richesse à cause des agissements de deux catégories d'acteurs : d'une part ceux qui, dans l'émission et la circulation des monnaies, possèdent un pouvoir notable comme les trésoriers, officiers des monnaies, usuriers, changeurs, affineurs, orfèvres et marchands ainsi que ceux dont l'action est exclusivement frauduleuse comme les faussaires, rogneurs et billonneurs (Bodin 1568, p. 141 ; Bodin 1593, VI/3, p. 121 et 134-136, etc.), et d'autre part les princes eux-mêmes, influencés par les flatteurs et courtisans qui leur «font hausser et rabaisser le pied des monnoyes » (Bodin 1568, pp. 130 sq). En effet, le support métallique des pièces, qui ne portent pas mention d'unité de compte, est souvent aisément falsifiable ou rognable. Les abus sont tels, tant de la part des souverains que des falsificateurs privés de tous ordres, que la question monétaire est très fréquemment abordée à partir de cette problématique ${ }^{6}$. Bodin ne fait pas exception et s'insère naturellement dans tout ce mouvement d'idées qui pourfend, particulièrement depuis le XIVe siècle et pour encore longtemps, la mauvaise gestion des princes, les abus des officiers de la

\footnotetext{
5. Il cite la livre de Paris (parisis), pourtant dans un rapport fixe à la livre tournois, mais aussi la livre de Bretagne et la livre de Normandie (Bodin 1593, VI/3, pp. 129-130).

${ }^{6}$. Voir l'ouvrage plus large de Sargent et Velde (2003, pp. 64-68 notamment), qui aborde la question à partir du problème des espèces de faible montant.
} 
Monnaie ou le crime des particuliers ${ }^{7}$. C'est donc par et contre la fausse monnaie que Bodin aborde les questions monétaires.

Dans ce contexte, la monnaie se conçoit au fond de deux manières : positivement, elle est la création de la loi du souverain, et négativement, elle n'est pas la fausse monnaie. Bodin propose une réforme monétaire radicale qui devrait conduire à faire s'imposer définitivement la conception positive de la monnaie en empêchant toute falsification monétaire.

Mais, pour l'heure, la fausse monnaie est une réalité contre laquelle il faut lutter. Lorsque, en 1568, Bodin répond aux Paradoxes de Malestroit, il commence par réfuter son argumentation en montrant que les prix, contrairement à ce qu'affirme son adversaire, ont vraiment augmenté. Il annonce trois causes à cet enchérissement: l'abondance d'or et d'argent (provenant notamment du nouveau monde), les monopoles et la disette; mais il en développe une quatrième : le plaisir des rois, qui pousse à la hausse le prix des choses qu'ils aiment. La plus importante de ces causes, affirme-t-il, est la première. La cause étudiée par Malestroit semble absente : Bodin affirme que «ce n'est pas pour avoir alteré les monnoyes que tout est encheri » (Bodin 1568, p. 113) car, même si cette altération (mutation nominale) a eu lieu, et il s'emploie tout d'abord à la minimiser, elle a été bien inférieure à la hausse des prix. La dernière partie de sa Response revient pourtant sur les raisons données par Malestroit et qu'il a du mal à réfuter catégoriquement. Elle est particulièrement consacrée à expliquer comment le système de monnaies doit être transformé pour stopper la falsification, seule façon selon lui «d'arrester à peu pres l'estimation et pris des choses »(Bodin 1568, p. 130). Il reconnaît de la sorte, malgré son intention première, que l'altération des monnaies est un grave problème.

En 1578, dans la révision de la Response sous le titre de Discours, il annonce " quatre ou cinq causes» de l'enchérissement des choses et développe cette fois explicitement comme cinquième cause la question du "pris des monnoyes» (Bodin 1578, p. 84). La dernière partie du texte est considérablement renforcée ; elle fait écho au chapitre sur les monnaies présent dans la République. La pensée de Bodin a eu le temps de mûrir ${ }^{8}$ et elle ne s'oppose plus que par effet de rhétorique à Malestroit sur la question de l'altération des monnaies comme cause de l'enchérissement des prix, au point que celle-ci devient centrale et éclipse presque la mise en évidence d'une relation entre l'abondance de métaux précieux et les prix 9 . On peut certes

${ }^{7}$. Jean Buridan, Bartole, Nicole Oresme, Nicolas Copernic, Malestroit, par exemple, précèdent Bodin dans la condamnation de l'altération des monnaies par les princes, avec diverses nuances.

${ }^{8}$. Ce qui n'empêche pas Bodin de commettre un certain nombre d'erreurs de citations, que Budruss (1987) interprète comme le résultat d'un manque de temps dans la rédaction du Discours.

9. Dans le Discours, la partie qui traite des moyens de remédier à l'enchérissement des choses, et qui est centrée sur la problématique de la fausse monnaie et le système idéal pour l'annihiler, 
interpréter avec Harsin (1928) et Gonnard (1935) ce fait étrange comme le résultat de la contradiction entre l'observation par Bodin de l'afflux de métaux précieux comme cause de l'inflation et l'idée commune à laquelle il semble souscrire selon laquelle l'abondance de métaux précieux est signe de richesse. On peut privilégier une autre interprétation, selon laquelle Bodin ne semble plus voir autrement que comme un argument de moindre portée ce que les économistes retiendront de son œuvre, précisément parce que ce n'est pas là ce qui fait le plus écho à ses préoccupations centrales : le plus important est pour lui de développer une réflexion sur le rapport de la monnaie à la souveraineté.

A Malestroit qui mettait en avant la problématique de la dépréciation de l'unité de compte du fait des mutations nominales, et qui produit ce que l'on qualifierait aujourd'hui d'illusion nominale ${ }^{10}$, Bodin répond, non seulement en montrant que la hausse des prix n'est pas une illusion et qu'elle a pour origine, notamment, l'abondance des métaux précieux, dépassant par là même Malestroit, mais aussi en substituant à la problématique de l'augmentation des monnaies (par les mutations nominales) celle de la falsification des monnaies, c'est-à-dire en faisant de ce dépassement non pas l'abandon d'une piste mais son intégration dans un corpus plus large et compréhensif. Or cette substitution est pour partie une simple reformulation des choses.

La question n'est pas ici de réévaluer le quantitativisme de Jean Bodin ${ }^{11}$. Cependant, se replacer dans le contexte de l'époque permet de constater que l'opposition entre Malestroit et Bodin n'est pas celle d'un officier haut placé dont l'analyse est limitée et l'explication erronée, à un grand théoricien qui, d'une intuition géniale, défait son adversaire tout en jetant les bases d'une théorie moderne de la monnaie. Il s'agit bien plutôt de deux hommes plus ou moins éloignés des centres de décision en matière monétaire et dont les idées empruntent tout à la fois des chemins déjà rebattus et des sentiers presque vierges. Il apparaît assez clairement que c'est la Cour des monnaies qui dispose d'une influence déterminante dans les décisions royales sur la monnaie (Parsons 2003). Malestroit est un officier de la Chambre des comptes, cour souveraine comme la Cour des monnaies mais qui ne dispose pas de compétence particulière en matière monétaire. Le rapport de Malestroit sur le fait des monnaies, commandité par la Chambre des comptes pour le Roi Charles IX, se présente comme des paradoxes qui sont des raisons «fort

occupe $38 \%$ du texte, contre environ $12 \%$ pour ce qui concerne l'abondance de l'or et de l'argent.

10. Malestroit (1566, p. 60) écrit ceci : "Et ainsi l'encherissement que lon cuide estre maintenant sur toutes choses, ce n'est qu'une opinion vaine, ou image de compte sans effet ni substance quelconque ».

11. Les textes récents de O'Brien (2000) et Arestis et Howells (2002) fournissent à cet égard des arguments, qui certes ne vont pas dans le même sens. La position la plus correcte semble être 
esloignees de l'opinion du vulgaire » (Malestroit 1566, p. 55), c'est-à-dire des «opinions contraires à la commune » (Bodin 1568, p. 74) non par leur dénonciation des altérations monétaires (chemin rebattu) mais par leur découverte de l'illusion nominale produite par des phénomènes monétaires (sentier vierge). Bodin, par la suite, répond à Malestroit en revenant au chemin rebattu de l'inflation quotidiennement constatée mais emprunte le sentier presque neuf d'une relation entre abondance de monnaie et niveau des prix, et en dégage la piste pour de plus profondes interprétations. Bodin exagère l'importance de cet argument presque inédit par rapport aux autres causes de la cherté des choses, particulièrement l'altération des monnaies qui, dès la publication de la République en 1576, revient logiquement sur le devant de ses analyses monétaires.

Le point central du discours de Bodin se déplace donc, au fil de ses textes économiques, de cette relation quantitative vers la problématique de la fausse monnaie. La définition de celleci n'apparaît pourtant jamais. C'est en creux qu'il faut la chercher. La fausse monnaie, si l'on reprend tous les éléments progressivement exposés par Bodin, est alors une monnaie altérée, une monnaie sur le pied de laquelle les flatteurs du roi agissent, une monnaie qui ne permet pas d'arrêter l'estimation et le prix des choses, une monnaie qui entrave le commerce, une monnaie rognée, une monnaie contrefaite, une monnaie dont l'aloi est faible, etc. Pour éclaircir la question, il faut distinguer les trois éléments qui constituent les pièces : le support métallique, la marque du souverain et le cours légal. On peut dès lors identifier trois catégories de fausse monnaie, selon ce sur quoi porte la falsification.

Précisons les termes que nous retiendrons : la fausse monnaie de Bodin, terme générique de ce qui résulte d'une falsification, peut être une monnaie altérée, une monnaie dégradée ou une monnaie contrefaite ${ }^{12}$.

- La monnaie altérée a un cours légal supérieur à la valeur de son contenu métallique. Cet écart ne se réduit pas au seul brassage ${ }^{13}$. Il procède d'abord des conditions du seigneuriage lors de l'émission des pièces, mais il s'accroît au fil des mutations nominales, ou «altérations » selon les termes employés par Bodin. La monnaie altérée provient donc en particulier des manipulations princières sur le cours des monnaies. Mais elle peut aussi provenir des mutations réelles, ou

celle de Schumpeter (1983, I, p. 432-6) qui y voit davantage un «métalliste intransigeant» qu'un quantitativiste.

12. On peut rapprocher ces catégories des neuf manières de commettre le crime de lèse-majesté que Bazinghen (1764, vol. 1) recense près de deux cents ans plus tard, à ceci près que Bazinghen n'y inclut pas les abus des princes eux-mêmes.

13. Le brassage est un tarif prélevé par la Monnaie pour couvrir les frais de production hors coût du métal. 
manipulations princières sur le contenu métallique lui-même. La monnaie altérée s’oppose ainsi à un idéal de monnaie dont le cours légal serait aligné sur le contenu métallique, que celui-ci soit bon ou mauvais.

- La monnaie dégradée possède un contenu métallique non conforme aux ordonnances royales, quelle que soit la qualité métallique définie par celles-ci. Ce défaut peut avoir plusieurs origines : le détournement ou la mauvaise application par les officiers des monnaies de ces ordonnances ${ }^{14}$; les rogneurs par exemple qui prélèvent une partie du métal de la pièce ; ou encore l'usure naturelle de la pièce.

- La monnaie contrefaite est forgée par des particuliers non dépositaires du droit souverain de frapper monnaie ; elle s'oppose à la vraie monnaie, celle frappée sur ordre du souverain. La qualité de la monnaie là encore n’intervient pas, car on peut imaginer des seigneurs frappant de la bonne monnaie et considérés comme faux-monnayeurs par le prince (Bodin 1593, I/10, pp. 332-333). Mais le cas le plus courant reste celui de particuliers usurpant ce droit du souverain pour l'appât du gain et, en conséquence, émettant de fausses pièces de mauvaise qualité.

Il y a falsification dans tous ces cas de fausse monnaie (cf le tableau 1 récapitulatif), mais pas pour les mêmes raisons : mauvaise gestion et abus (monnaie altérée), crime (monnaie dégradée) voire lèse-majesté (monnaie contrefaite). Les criminels encourent des supplices (les faux-monnayeurs peuvent être rôtis et bouillis), les princes versés dans l'abus encourent "l'infamie de faux monnoyeur», comme Philippe le Bel (Bodin, 1593, VI/3, p. 118). C'est en ce sens que la Response de Bodin n'est pas en totale opposition avec les Paradoxes de Malestroit : les Paradoxes considéraient l'altération des monnaies comme la cause principale de l'illusion de hausse des prix, ce qui est une bonne moitié de ce que pourfend Bodin lorsqu'il cherche à « exterminer les faux monnoyeurs [et] descorner les flatteurs » (Bodin 1568, p. 130) $)^{15}$.

\section{La monnaie altérée et la monnaie dégradée à la racine de la fausse monnaie}

La fausse monnaie constitue donc le point central du discours monétaire de Bodin. Ce thème est loin d'être neuf, mais l'intérêt est qu'il prend place dans la conception bodinienne de la souveraineté.

De cette conception découle une hiérarchisation paradoxale des trois composantes de la fausse monnaie. D’un point de vue purement logique, la contrefaçon monétaire doit être autrement plus grave que la monnaie dégradée et a fortiori la monnaie altérée. La contrefaçon usurpe en effet la marque du souverain, elle correspond à ce que Bodin appelle un crime de lèse-

14. Bodin (1593, VI/3, p. 139) cite le cas de Pinatel vers 1552. 
majesté. La monnaie altérée et la monnaie dégradée, de leur côté, provoquent bien quelques conséquences néfastes pour la république : les mutations continues et la mauvaise qualité des pièces empêchent la fixation de repères stables en matière de prix, et donc d'activité marchande.

Il faut donc lutter contre la fausse monnaie, certes, mais alors Bodin renverse la hiérarchie : si la monnaie est un métal marqué par le souverain, le point essentiel est la qualité du métal, et non la marque. Bodin a en effet conscience du fait que le but de la contrefaçon monétaire (donc du fait de particuliers) n'est pas de contrefaire la marque, mais de réaliser un profit en contrefaisant le métal. Il se trouve que, pour un particulier, contrefaire le métal d'une monnaie nécessite de contrefaire la marque. En éliminant les caractéristiques du système de monnaies officiel qui rendent possible la contrefaçon du métal, le crime de lèse-majesté disparaîtra ; aussi les solutions qu'il expose pour parvenir à exterminer les faux-monnayeurs sont-elles essentiellement centrées sur la recherche d'une infalsifiabilité du contenu métallique des pièces.

La lutte contre la falsification de la marque du souverain (la monnaie contrefaite) est donc subordonnée à la lutte contre la falsification du métal (dans son sens de monnaie dégradée comme de monnaie altérée, celle-ci produisant un écart permissif entre cours légal et valeur intrinsèque). Du reste, Bodin, comme tout le monde à l'époque, sait que des monnaies de nombreuses origines circulent dans le royaume ${ }^{16}$. Malgré sa conception d'une souveraineté absolue, il ne considère pas que cette intrusion d'éléments étrangers contredise la souveraineté monétaire du prince. Il ne semble en effet s'inquiéter des monnaies étrangères que lorsque leur contenu métallique est d'un titre inférieur à celui des pièces du royaume, car alors cela signifie que les mauvaises pièces vont affluer et être converties en pièces françaises à leur tour exportées et fondues à l'étranger : selon l'adage attribué au XIXe siècle par McLeod à Thomas Gresham, la circulation de mauvaise monnaie, en l'occurrence étrangère, se substitue à celle de la bonne, en l'occurrence française. Enumérant les conditions d'un système de bonne monnaie et citant tous ses avantages, Bodin explique qu'il faudrait, pour éviter le fonctionnement de cette substitution, décrier les pièces étrangères d'aloi inférieur et interdire l'exportation des pièces françaises ; Bodin est conscient de l'inefficacité de cette dernière mesure, mais elle est nécessaire pour limiter un peu la fuite des - bonnes - pièces françaises, et elle n'annulerait pas l'attrait de leur possession, qui pousserait les marchands étrangers à faire commerce et serait donc bénéfique pour l'activité du royaume (Bodin 1568, p. 134 et pp. 137-138).

15. Les flatteurs en question sont ces courtisans qui poussent le prince à augmenter les monnaies.

16. En mai 1577, un édit de Henri III démonétise environ 180 types de pièces qui proviennent d'une vingtaine d'espaces de souveraineté différents, et il laisse circuler les pièces espagnoles seulement, unanimement considérées comme bonnes. Voir Parsons 2003. 
Qu'importe, donc, que les pièces en circulation en France soient émises par le roi ou par un prince étranger, pourvu que les pièces soient de qualité. On comprend que la marque n'est pas, pour Bodin, l'essence de la monnaie, entendue comme ce qui n'est ni monnaie contrefaite, ni monnaie dégradée, ni monnaie altérée : selon lui le seul élément qui doit importer est le métal fin dont la pièce est constituée. De plus, on le verra, Bodin préconise d'éliminer tout seigneuriage et tout brassage. Chaque pièce ne doit donc avoir pour valeur que celle du métal qui la porte, la marque ne possédant aucune valeur en elle-même ou n'ayant aucune incidence sur la valeur de la pièce. Ainsi son point de vue est-il exclusivement métalliste.

La logique de Bodin est donc celle de l'étalon et, poussée à bout, elle le substitue à l'unité de compte. Bodin peut en effet ignorer presque totalement l'existence de l'unité de compte puisque, dans le système idéal auquel il aspire, elle n'est rien d'autre qu'une unité de poids. Ce métallisme le conduit ainsi à ne pas tant se focaliser sur les pièces (métal + marque + cours légal) que sur le seul métal. De là la prééminence de la problématique de la fausse monnaie, sous son acception de monnaie dégradée et de monnaie altérée plus encore que de monnaie contrefaite. C'est ce triple sens de la fausse monnaie qu'il convient maintenant d'approfondir en le reliant au système juridique de Bodin.

\section{Du droit souverain à la foi publique, ou la nécessité d'une monnaie juste}

Ce système juridique permet à Bodin d'explorer le «droit gouvernement» de la république et ce » qui la fonde, c'est-à-dire la «puissance souveraine» du prince (Bodin 1593, I/1, p. 27). Quelle place l'auteur donne-t-il à la question monétaire dans le vaste système de pensée de la République qui est avant tout un traité juridico-philosophique? Afin de limiter dans son exercice le pouvoir monétaire du prince, Bodin est amené à distinguer en matière monétaire deux niveaux d'obligations : la loi (A) et le contrat ou convention (B).

\section{A. Le droit de battre monnaie est de même nature que la loi}

La république se définit par une puissance souveraine et un ensemble de choses publiques. La monnaie est, dans cet ordre d'idées, assez loin des préoccupations centrales de Bodin. Il ne la mentionne même pas de façon directe parmi ces choses qui font que l'on peut parler de république ; elle n'apparaît que sous l'angle des finances :

« le domaine public, le thresor public, le pourpris de la cité, les rues, les murailles, les places, les temples, les marchés, les usages, les loix, les coustumes, la justice, les loyers, les peines, et autres choses semblables, qui sont ou communes, ou publiques, ou l'un et l'autre ensemble : car ce n'est pas Republique s'il n'y a rien de public » (Bodin 1593, I/2, pp. 4344). 
Cet absence n'est au fond pas surprenante ; elle tient à ce que Bodin considère la monnaie sous l'angle de la frappe, qui procède de la puissance du roi seul (même s'il peut déléguer l'opération du monnayage) plutôt que sous l'angle de la circulation, dans laquelle elle serait nécessairement considérée comme publique. C'est donc ailleurs qu'il faut la chercher.

C'est lorsqu'il définit la souveraineté et ses marques qu'apparaît explicitement la monnaie. La souveraineté est définie comme "la puissance absolue et perpétuelle d'une république 》 (Bodin 1593, I/8, p. 179) ; cette puissance absolue est cela même qui fonde toute république : il s'agit de «l'autorité suprême en quoi réside le principe de la République» (Bodin 1566, p. 158). La puissance souveraine du prince a des marques qu'elle seule possède. La première de toutes, et qui fonde toutes les autres, est la puissance de donner lois aux sujets, lois auxquelles le souverain est seul à ne pas être soumis (Bodin 1593, I/10 p. 306) ${ }^{17}$. Il n'est en effet soumis qu'à la loi divine et naturelle, qui est celle de l'Ancien Testament, et qui dicte ce qui est juste. Mais si la loi de Dieu freine la souveraineté, ce n'est pas la souveraineté dans son principe mais dans son exercice qui s'en trouve limitée. Car, de façon générale, la souveraineté est absolue dans son principe mais pas dans son exercice ; aussi n'incline-t-elle pas à l'absolutisme (Goyard-Fabre 1989, pp. 159-164 et Mairet 1993). D’un point de vue normatif, les lois du souverain devraient donc avoir pour seule et unique fin la justice divine (Bodin 1593, I/8, p. 228). De cette première marque de la souveraineté découlent toutes les autres, dont celle de «hausser ou baisser le titre, valeur et pied des monnaies» (Bodin 1593, I/10, p. 309). Cette marque de souveraineté apparait parmi un ensemble de marques qui, certes, découlent de la première, mais elle est absente des cinq que détaille explicitement Bodin par la suite. Mieux : dans la Méthode de l'bistoire, ouvrage antérieur à la République, ce n'est pas cette manipulation des monnaies qui apparaissait parmi ces marques de souveraineté un peu marginales, mais le droit de battre monnaie (Bodin 1566, pp. 158-159).

Que peut-on conclure de cette évolution et de la position finale de Bodin qui est celle de la République ? On doit d'abord comprendre que la monnaie en elle-même n'est pas une marque de la souveraineté : le propre de la souveraineté est le pouvoir de modifier le cours légal et le contenu métallique des pièces. Mais si cette manipulation relève de la puissance de la loi, qui est celle du souverain, seul le droit de battre monnaie est de la même nature que la loi ${ }^{18}$. Ainsi

17. Le premier ouvrage de Bodin, La méthode de l'bistoire, mentionnait cette fonction de législation seulement comme la seconde marque de la souveraineté (Bodin 1566, pp. 158-159), tandis que la République lui donne le premier rôle, de surcrôt fondateur de toutes les autres marques.

18. "Quant au droit de moneage [battre monnaie], il est de la mesme nature que la loy ", écrit Bodin (1593, I/10, p. 331). 
l'origine de la monnaie est-elle identifiée dans la loi, comme l'indique son nom d'origine grecque, nomos (Bodin 1593, I/10, p. 331) ${ }^{19}$.

Battre monnaie est donc placé au même niveau que la loi du souverain. Aussi, la frappe illicite de monnaies est une usurpation de cette loi et un crime de lèse-majesté, que le contenu métallique de ces monnaies soit bon (titre - ou aloi ou loi - élevé, c'est-à-dire l'or à vingt trois carats et l'argent à onze deniers douze grains) ou mauvais (titre plus faible). Ainsi, dans notre catégorie triptyque de la fausse monnaie, la monnaie contrefaite relève-t-elle d'une usurpation de la loi (cf tableau 2). Lorsque le souverain octroie des privilèges à certains seigneurs afin qu'ils puissent battre monnaie sur leur domaine, ou lorsqu'il confie à des officiers des monnaies la charge de frapper monnaie dans diverses villes du royaume, il reste néanmoins seul maittre du cours légal et du contenu métallique des pièces, et contrôle par ailleurs les marques que l'on appose sur ces pièces. Bodin souhaite de toute façon éliminer ces restes de féodalité ainsi que la multiplicité des ateliers, comme le fit, sans suite, François Ier, et n'instaurer qu'un seul maître des monnaies, dans un seul atelier monétaire pour tout le royaume (Bodin 1593, VI/3, pp. 135138). Il s'agit de centraliser le monnayage pour mieux concentrer le pouvoir monétaire dans les mains du souverain dont il émane par définition et mieux contrôler les abus des officiers des monnaies conduisant à une monnaie dégradée.

Cette distinction qu'opère progressivement Bodin entre l'acte de battre monnaie et l'acte de manipuler ses caractéristiques (métal et cours légal) parait à la fois artificielle et ambiguë. Il y a certes deux opérations différentes mais elles ne se distinguent pas ainsi. Battre monnaie est l'occasion de jouer sur le contenu métallique, tandis que l'établissement d'un cours légal est une tout autre opération, beaucoup plus souple et plus fréquente.

Une explication possible de cette évolution et de l'ambiguïté qu'elle produit tient à son constat de la nature pernicieuse et perverse des mutations réelles. On peut alors comprendre que Bodin cherche à ne pas soumettre ces actes à la seule loi du prince. Cette loi, affirme-t-il, a puissance souveraine, donc ne regarde personne d'autre que le prince lui-même, sous le regard de Dieu. Le seul regard divin est bien impuissant à freiner les dérives ici-bas, et on peut comprendre que ce n'est pas pour Bodin un frein suffisant à l'exercice de la puissance de la loi : le prince peut toujours justifier la falsification des monnaies par son droit royal. L'évolution de la pensée de Bodin de la Méthode de l'bistoire à la République peut donc s'interpréter ainsi : la seule façon que trouve Bodin de limiter le pouvoir du prince dans le domaine monétaire est de lier non plus à la

\footnotetext{
19. Voir aussi Bodin (1568, p. 143-144) où il est écrit que « la monnoye est une loy à bien parler».
} 
loi, mais au contrat, l'acte d'altérer le contenu métallique des pièces, l'extrayant ainsi de l'acte de monnayage, et l'analysant à l'instar des modifications de cours légal20.

\section{B. La valeur et le titre des monnaies sont de l'ordre du contrat}

A la différence de la contrefaģon monétaire qui est de l'ordre de la transgression de la loi et du crime de lèse-majesté car elle empiète sur le droit exclusif du souverain, la monnaie altérée du fait du prince ne relève donc pas de la loi, mais du contrat (cf tableau 2). Comment Bodin introduitil cette notion, quel lien peut-on faire avec la monnaie, et en quoi cela constitue-t-il une solution à l'impasse à laquelle se heurtait Bodin?

La différence entre le contrat (aussi appelé convention) et la loi réside dans les obligations qui en résultent pour le prince :

«Il ne faut donc pas confondre la loy et le contract : car la loy depend de celuy qui a la souveraineté, qui peut obliger tous ses subjects, et ne peut s'y obliger soy même : et la convention est mutuelle entre le prince et les subjects, qui oblige les deux parties reciproquement : et ne peut l'une des parties y contrevenir au prejudice, et sans le consentement de l'autre et le prince en ce cas n'a rien par dessus le subject » (Bodin 1593, I/8, p. 195).

L'obligation réciproque qui naît du contrat ou de la convention est d'une part, pour les sujets le devoir de «foi, sujétion, obéissance, aide, et secours» envers le prince, et d'autre part, pour le prince, le devoir de "maintenir par la force des armes et des lois ses sujets en sûreté de leurs personnes, biens, et familles » (Bodin 1593, I/7, p. 151). Au total, le prince leur doit la justice. Cette justice consiste notamment dans le respect de ses conventions ou contrats,

« car puisqu'il [le prince] est garant aux sujets des conventions et obligations mutuelles qu'ils ont les uns envers les autres, à plus forte raison est-il débiteur de justice en son fait (...). L'obligation est double : l'une pour l'équité naturelle, qui veut que les conventions et promesses soient entretenues, l'autre pour la foi du prince, qu'il doit tenir, [alors] qu'il y eut dommage, parce qu'il est garant formel à tous ses sujets de la foi qu'ils ont entre eux.

(...) Dieu même, comme dit le maître des sentences, est tenu de sa promesse » (Bodin 1593, I/8, pp. 218).

C'est donc justice et vertu pour le prince que de respecter ses contrats, non seulement parce qu'il tient sa promesse, mais aussi parce qu'il donne l'exemple aux sujets. De là dérive

20. Ainsi, de 1566 à 1576, Bodin suit en matière monétaire un cheminement opposé à celui de sa conception de la souveraineté, pour laquelle il passe à conception absolutiste (voir Franklin 1993 pour ce cheminement). 
toute la problématique de la foi publique, essentielle en matière monétaire, que Bodin n'aborde pas dans sa Réponse mais seulement dans la République puis dans le Discours.

La question de la manipulation du titre et du cours légal des monnaies rejoint au fond celle des abolitions de dettes dans la mesure où se présente le danger d'une "entière éversion d'un Etat» dès lors que "les obligations sont cassées, les contracts annullez, les debtes abolies »: "il n'y aura fiance quelconque de l'un à l'autre ». Car « la foy gist aux promesses des conventions légitimes » (Bodin 1593, V/2, p. 62). Il est aisé de voir là des références aux banqueroutes, intervenues pour les finances royales françaises en 1558 puis 1567-1568, ou pour celles espagnoles en 1557 et 1575 . Rompre de la sorte la foi publique est en effet s'assurer que plus aucune convention ou contrat ne pourra se passer puisque l'exemple offert par le prince montre qu'on ne peut avoir confiance en des paroles données. C'est l'occasion pour Bodin (ibid.) de remarquer que les républiques n'ont pas pour fondement l'égalité des biens mais la foi, et qu'en conséquence les abolitions de dettes, même mises en oeuvre afin de réaliser cette égalité, n'ont que de funestes effets puisque la foi se trouve rompue. Mieux, l'égalité est source d'inimitiés et de séditions. Au total, l'égalité doit bien se distinguer de la justice : celle-ci réside non pas dans la recherche d'une illusoire et dangereuse égalité, mais dans l'application par le prince de la loi naturelle et divine. La justice est équité, non égalité.

La question du titre et du cours légal des monnaies ne relève pas de la notion d'égalité, mais bien de celle de justice. Or ce sont les tromperies des courtisans et flatteurs du prince qui conduisent celui-ci à manipuler le titre et le cours légal des monnaies. Ces manipulations ne profitent ni au prince, ni au peuple, mais seulement à ceux qui sollicitent ou sentent la venue de ces manipulations :

« les uns prestent à grand interest le billon, \& puis trouvent moyen de le faire decrier, pour estre payé en forte monnoye : les autres acheptent à vil pris le billon decrie, car le peuple est contraint de le vendre au plaisir des changeurs \& maistres des monnoyes, si on ne le vend au marc. Les autres empruntent de tous costez, ayant senti le vent qu'on veut hausser la valeur des monnoyes : ou bien eux mesmes sollicitent les princes à ceste fin » (Bodin 1568, p. 141).

Ces manipulations sont l'instrument d'une «injustice barbaresque »(Bodin 1568, p. 144)21. Altérer la monnaie en diminuant son titre ou en augmentant son cours rompt une convention

21. Il existe cependant une circonstance dans laquelle la baisse du titre ou la hausse du cours légal serait juste : la nécessité. Cette nécessité est celle des finances; on peut donc considérer comme juste la manipulation des monnaies lorsqu'il est nécessaire de lever des fonds pour faire face à des dépenses non ordinaires. Bodin ne précise cependant pas sa pensée quant à ces dépenses ; il constate simplement que l'on n'a, dans les faits, jamais manipulé les monnaies par nécessité, et qu'au contraire, lorsque Charles VII eut un tel besoin, il s'attacha à frapper une bonne monnaie, 
tacite entre le prince et ses sujets. Dès lors que le prince se dédit, comment les sujets pourraientils se sentir encore liés par ce contrat, comment pourraient-ils encore considérer ce prince comme digne de "fiance»? Ce prince devient, à l'instar du roi Philippe le Bel dont l'affaiblissement des monnaies qu'il ordonna en 1300 provoqua de grands troubles dans le royaume, un roi faux-monnayeur, "falsificatore di moneta », dit Dante (Bodin 1593, VI/3, p. 118). Voilà donc la solution de Bodin pour limiter l'exercice de la souveraineté en matière monétaire : confier au domaine du contrat la valeur et le titre des monnaies, et montrer que la rupture de ce contrat est des plus dangereuses. Ainsi, marque de souveraineté, le droit du prince de manipuler le titre et la valeur des monnaies doit cependant s'arrêter là où commence l'injustice et l'abus. C'est tout le coeur de l'analyse monétaire de Bodin : le prince, en toute logique, possède le droit de falsifier les monnaies, mais au nom de préoccupations théologiques (la loi divine et naturelle), et surtout de préoccupations morales (la justice et la foi publique), il est préférable qu'il ne le fasse pas. Cette construction dérive du caractère du contrat. Il reste que la vertu du prince demeure le dernier facteur de décision pour que le système de monnaies idéal qui est censé contrecarrer toute falsification soit efficace. Ce n'est pas suffisant ; aussi Bodin va-t-il tenter de contraindre le souverain à cette vertu.

\section{La vertu contrainte du souverain}

Il s'agit pour Bodin de limiter concrètement le pouvoir monétaire du prince. Il souligne le risque de rompre la foi publique en violentant les coutumes, mais ceci n'apparaît pas suffisant (A). La proposition d'un système idéal de monnaies doit permettre de lier les mains du souverain (B), mais en dernière instance seule une pression étrangère semble à même de limiter l'exercice de la souveraineté monétaire (C).

\section{A. La foi publique contre la loi du souverain}

Si le souverain fait la loi et l'impose à tous ses sujets, s'il doit tenir ses conventions pour entretenir la foi publique, les sujets font, eux, la coutume.

«Si le prince souverain est maittre de la loi, les particuliers sont maitres des coutumes. (...) La coutume prend sa force peu à peu, et par longues années d'un commun consentement de tous, ou de la plupart (...). La coutume se coule doucement, et sans force (...). La

et ne l'altéra que plus tard, alors que son royaume était revenu à la paix (Bodin 1568, pp. 136137). Dans d'autres circonstances que la nécessité, Bodin récuse que la manipulation des monnaies soit un bon moyen de lever des fonds. Lors des Etats généraux de 1576-77, auxquels Bodin participe et dont il donne un récit, il ne propose d'ailleurs pas une telle méthode alors même que la question du financement d'urgence est posée directement par le roi à l'assemblée du tiers état et qu'il a un rôle important dans les délibérations du tiers (Bodin 1577). 
coutume n'a force que par la souffrance, et tant qu'il plaît au prince souverain, qui peut faire une loi »(Bodin 1593, I/10, pp. 307-308).

Bodin raisonne donc sur l'écart existant entre le droit écrit et le droit coutumier ${ }^{22}$. Or si la coutume «n'a pas moins de puissance que la loi» (Bodin 1593, I/10, pp. 307-308), elle ne doit pas pour autant déroger à la loi du souverain, car celle-ci, qui est «commandée et publiée par puissance, et bien souvent contre le gré des sujets », domine la coutume. Définie dans son émergence et dans sa pérennité, la coutume n'est pas pour autant définie dans son contenu.

Il existe pourtant une incertitude dans la formalisation de Bodin. Certes, la coutume trouve sa force dans son lent enracinement: "la coustume prend sa force peu à peu, et par longues annees d'un commun consentement de tous, ou de la plus part» (Bodin 1593, I/10, p. 307). La loi au contraire trouve sa force dans la puissance du souverain. Certes, donc, la loi peut casser les coutumes, mais Bodin craint de trop les changer, surtout de façon violente, précisément de peur de briser la foi publique, d'où le souverain tire sa force ${ }^{23}$.

La pensée de Bodin aboutit de la sorte à deux affirmations avortées : premièrement, la frappe des monnaies est de l'ordre du droit du souverain, mais ce droit est limité, dans la définition du contenu métallique des monnaies, par l'aspiration du souverain à respecter ses engagements et justes conventions ; secondement, la loi l'emporte sur la coutume, mais cette puissance est limitée par la nécessité de sauvegarder la foi publique. En l'occurrence, foi publique et respect des monnaies par le souverain vont de pair. La loi, qui, dans son principe, a une puissance sans limite dans la république, est donc concrètement limitée à la fois par la promesse que le souverain doit respecter lorsqu'il s'engage par une convention ou contrat, et par la nécessité de ne pas trop violenter les coutumes. Goyard-Fabre souligne le renversement qu'opère Bodin : puisque le souverain n'est tenu, d'un point de vue juridique, par aucun serment, c'est d'un point de vue moral, par la bonne foi, que Bodin veut l'obliger.

«Tenir ses engagements est donc une règle morale plutôt qu'une règle juridique. Et, derrière cette règle morale, c'est la loi divine qui s'impose comme règle d'ordre, de droiture et de vertu » (Goyard-Fabre, 1989, pp. 95-96).

Mais l'histoire ne montre guère l'efficacité des règles morales en matière monétaire. Qui plus est, aux falsifications du fait du prince s'ajoutent les falsifications du fait des particuliers

\footnotetext{
22. La France s'est progressivement divisée, à partir du XIIe siècle, en pays de droit écrit (romain), au sud, et pays de droit coutumier, au nord. Charles VII enclenche en 1454 un processus long d'écriture du droit coutumier. Progressivement, le Parlement de Paris acquiert un ascendant sur l'évolution de ce droit. Bodin, né à Angers en pays de droit coutumier, est formé au droit à Toulouse, pays de droit écrit, puis tente, en vain, de devenir avocat à Paris. C'est après cet échec qu'il se consacre en particulier à l'étude du droit et de la philosophie.
} 
comme le billonnage et le rognage des monnaies, sans parler des contrefaçons ${ }^{24}$. C'est donc finalement pour stopper l'usurpation de la loi par les sujets et la rupture des justes conventions par le prince que Bodin propose un système idéal de monnaies.

\section{B. Un système idéal de monnaies}

Ce système, présenté dans la Réponse de 1566, est complété dans la République en 1576 et le Discours de 1578. La version de 1578 comporte les recommandations suivantes (livre VI, chapitre 3):

- La racine des abus est le mélange entre les métaux or, argent et cuivre, ce que Bodin appelle leur "confusion», "Laquelle cessant, ni le subject ni l'estranger n'y pourra faire aucune fraude, qui ne soit aussi tost descouverte » (Bodin 1593, VI/3, p.

135). Il faut dès lors supprimer les monnaies de billon (alliage pauvre de cuivre et d'argent). Si c'est possible, il faut aussi supprimer les monnaies de cuivre, car leur valeur est trop variable ${ }^{25}$. Au total, il faudrait que ne subsistent que des monnaies d'or et des monnaies d'argent. Pour remplacer les pièces de faible valeur, il faut frapper des petites pièces d'argent, jusqu'à un minimum de $1 / 4000$ de marc d'argent ${ }^{26}$.

- Les monnaies d'or et d'argent doivent avoir le titre élevé et immuable de 958\%, c'est-à-dire un titre de 23 carats pour l'or et 11 deniers 12 grains pour l'argent ${ }^{27}$.

- Les pièces doivent avoir un cours légal immuable, et ce cours légal doit correspondre exactement à leur valeur intrinsèque, le seigneuriage et le brassage étant abolis ${ }^{28}$.

23. Les républiques «n'ont appuy ni fondement plus asseuré que la foy, sans laquelle ni la justice, ni societé quelconque ne peut estre durable» (Bodin 1593, V/2, p. 62).

24. Le billonnage est un crime puni au même titre que le faux-monnayage. Il consiste en particulier à profiter de sa situation de pivot dans l'activité économique (changeur, orfèvre, marchand...) pour acheter certaines espèces de mauvais aloi à leur valeur intrinsèque pour les changer ensuite au cours légal contre des monnaies de bonne qualité, ou à remettre en circulation des espèces décriées ou dégradées.

25. Si Bodin rejette clairement le billon (en particulier car il est fondu pour récupérer l'argent qu'il contient), il hésite sur le cuivre. L'élimination de la monnaie de cuivre est davantage un souhait qu'une exigence ; il hésite entre des petites monnaies de cuivre ou d'argent, puis affirme sa préférence pour l'argent.

26. Comme la pièce de valeur la plus élevée qu'envisage Bodin est une pièce d'or de 1/16 de marc, on obtient une échelle de valeur de 1 à 3000 entre la plus petite et la plus importante des pièces. Cette échelle apparait supérieure à celle en cours à l'époque de Bodin. La plus petite des pièces correspondrait à un peu moins d'un denier de l'époque.

${ }^{27}$. La finesse de l'or s'exprime en 24 carats et celle de l'argent en 12 deniers.

28. Pour financer le brassage, Bodin préconise l'instauration d'une taille. 
- Le ratio entre la valeur de ces métaux doit être fixé définitivement à un pour douze. Ce taux résulte, selon Bodin, d'une moyenne sur plusieurs décennies du ratio or/argent autour de laquelle il varie fort lentement ${ }^{29}$.

- L'opération de monnayage doit être concentrée en un seul hôtel des monnaies, dans une seule ville du royaume.

- On doit retourner à la technique du moulage, plutôt que la frappe au marteau ${ }^{30}$.

Ce système doit être à l'épreuve de toutes les formes de fausse monnaie. Le moulage doit donner des pièces de meilleure qualité et plus difficiles à contrefaire; la concentration de la frappe monétaire doit permettre de mieux contrôler et circonvenir les abus des officiers des monnaies conduisant à une monnaie dégradée ; la règle du cours légal immuable et aligné sur le contenu métallique doit empêcher le souverain d'altérer les monnaies en haussant leur cours; la pureté des pièces doit conduire tout un chacun à repérer aisément les contrefaçons; etc. Au total, chacun, jusqu'aux "plus grossiers et ignorans», doit pouvoir immédiatement reconnaître la qualité des pièces en faisant simplement appel à ses sens : «à l'œil, au son, au poids, sans feu, sans burin, sans touche »(Bodin 1593, VI/3, p. 124)31. Reconnaitre le vrai du faux et le bon du mauvais étant aisé, cela ne pourra que réduire les abus des uns et des autres si ceux-ci sont surveillés par tous.

Il reste que ce système va au-delà de la seule lutte contre les monnaies altérées, dégradées et contrefaites car la contrainte des métaux purs conduit à proposer une "bonne monnaie» radicalement nouvelle. Pour le dire autrement, on peut avoir une monnaie non dégradée, non altérée et non contrefaite mais dont la finesse n'atteint pas des sommets ; Bodin souhaite qu'elle les atteigne (le tableau 1 positionne cette bonne monnaie vis-à-vis des autres formes de monnaie).

Ce système idéal est fait pour être commode, cohérent et stable, rompant avec les subtilités du système monétaire en vigueur qui ont pour effet de soumettre la plupart de la

\footnotetext{
29. Ce taux se justifie aussi par sa connotation alchimique (rapport lune/soleil par exemple) et la place qu'il tient dans la vie quotidienne (1 sou représente 12 deniers; la base 12 est une base simple, divisible par deux et trois).

30. Si Bodin cherche à supprimer la frappe au marteau, sa position évolue. En 1568, il propose l'usage du moulin, ou balancier, technique mise en œuvre en Allemagne dès 1550 et qui fera l'objet de refus obstinés de la Cour des monnaies jusqu'en 1645. A partir de 1576, Bodin critique cette technique et propose le moulage (Bodin 1593, VI/3, p. 143).

31. La «touche» dont il est question est la pierre de touche, un instrument que seuls les professionnels du maniement d'argent possèdent et qui exige de connaître la variété des couleurs qu'une pièce rend sur la pierre pour déterminer la qualité métallique de la pièce.

Il parle aussi de la facilité de reconnaissance de la qualité des pièces dans sa Response, mais elle n’apparaît pas aussi évidente (Bodin 1568, p. 130).
} 
population à ces pivots et spécialistes de la circulation monétaire que sont les changeurs, orfèvres, marchands, etc. (Blanc 1994). En somme, la finalité de Bodin est d'établir une convention mutuelle stable parce qu'immuable entre le prince et les sujets. La nature de cette convention est dictée par la nécessité d'éviter toute falsification, de quelque origine qu'elle soit. Les composantes du système idéal doivent le permettre. L'établissement d'un tel contrat apparaît à Bodin comme la seule et unique solution au regard de son contexte historique. Il constate en effet que, quelle que soit la valeur donnée par la loi aux monnaies, la prise effective des pièces se fait à un cours volontaire, résultant soit d'une valeur de convention entre différents acteurs, soit sur la base de la valeur métallique. Ces deux possibilités reposent en réalité sur le pouvoir des pivots de la circulation monétaire d'imposer de tels cours à l'ensemble des acteurs de la circulation des biens. Dès lors, le seul point d'ancrage juste qui s'offre pour repenser le système est la valeur métallique intrinsèque des pièces. L'établissement d'un tel contrat apparaît aussi comme la seule et unique solution pour sauvegarder la foi publique, gouverner les monnaies selon la justice naturelle et divine, et éliminer ces pratiques honnies de rognage, de billonnage et de falsification en général.

\section{Lier les souverainetés, solution de l'aporie de Bodin?}

Le métallisme de Bodin est ainsi profondément ancré dans une analyse réaliste de la constitution, des racines et des errements du système monétaire. Réfléchi et raisonné, ce métallisme devient normatif lorsque l'auteur propose son système idéal : le recours à l'étalon comme base absolue du système de monnaies est perçu comme la seule façon de reconstruire un système viable et à l'abri des tentations - fussent-elles celles du prince ou celles des particuliers. Sa proposition, formulée en 1568 puis reformulée en 1576, intervient dans un contexte de profonds bouleversements monétaires et de débats qui aboutissent aux réformes de 1577 (principalement l'abandon du compte en livre et sous, unités de compte abstraites, au profit d'un compte en écus, matérialisés par des pièces; voir Parsons 2003). Bodin affirme avoir présenté une version de son projet lors d'une réunion à laquelle il a participé durant les Etats généraux de Blois avec le surintendant des finances, le président de l'assemblée du tiers état, dont il était député du Vermandois, et trois généraux des monnaies ; il a été considéré comme impraticable ${ }^{32}$. Les mesures prises plus tard dans l'année 1577 l'ont été indépendamment des propositions de Bodin.

32. Bodin (1593, VI/3, p. 144) parle de cet échec. Bodin de Saint-Laurent (1907, p. 171, qui ne fait guère preuve de distance et de pertinence à l'égard de son ancêtre), Harsin (1928, p. 43, reprenant Bodin de Saint-Laurent) ou Parsons (2003, p. 7) évoquent cet échec, mais il semble y avoir un malentendu car il apparait clairement dans le récit des Etats généraux que fait Bodin 
La construction idéale de Bodin souffre d'un problème central : sa réussite suppose que le prince ne soit pas tenté d'altérer ses monnaies. S’il cède à la tentation, en effet, il ouvre la voie à la contrefaçon privée et aux abus de tous ordres. Il est hasardeux de croire que le prince sera suffisamment vertueux pour éviter cela. Bodin est donc conduit à tempérer le caractère absolu de la souveraineté en imposant au prince une série d'arguments pour le convaincre de ne point falsifier. Il s'agit d'être vertueux et juste (respecter la loi naturelle et divine) et de respecter la foi publique sur laquelle s'enracine le pouvoir du prince en respectant ses propres conventions, étant entendu que la stabilité des monnaies (dans la réalité) et, mieux, la coïncidence de leur cours légal avec leur contenu métallique à la finesse maximale (dans le système idéal) doivent constituer l'une de ces conventions. Mais cela ne peut être efficace contre la fausse monnaie que si le prince reconnait comme valables et justes ces limites. Ce qui est poser une condition philosophique et morale à l'exercice du pouvoir, qui peut fort bien s'en passer.

Le seul élément qui puisse, dans les faits, contraindre le prince à renoncer à falsifier les monnaies peut être trouvé dans un passage qui, à vrai dire, surprend par sa modernité. L'idée apparaît dans la République, en 1576. Elle est courte, rapidement passée, mais elle est, semble-til, la clé du système de Bodin. Il s'agit de généraliser à l'ensemble des royaumes voisins (on pense à l'ensemble de la Chrétienté) ce système idéal, de sorte que chacun soit lié aux autres. Cette généralisation ne peut être menée que par un grand prince dont la puissance permettrait de faire entendre cette idée ; il ne s'agit pas pour autant de renier la souveraineté de chacun de ces princes, mais de les lier par leur «commun consentement», selon un nouveau type de convention, par le biais de leurs ambassadeurs respectifs (Bodin 1593, VI/3, pp. 140-141)33. On peut trouver au moins deux avantages à une telle généralisation du système idéal. Tout d'abord, nul souverain ne craindrait plus l'intrusion de la mauvaise monnaie du voisin puisque toutes seraient identiques, à la marque de souveraineté près. Ensuite, chacun se garderait bien de rompre le cercle vertueux par crainte des répercussions sinon internes (troubles sociaux, émeutes, jacqueries...), du moins externes : Bodin montre bien que la fausse monnaie, et parfois la

que ce projet n'y a pas été évoqué (Bodin 1577). La proposition a donc eu lieu en marge des Etats généraux; la présence des officiers de la Cour des monnaies, ayant une autre conception des choses et un poids déterminant dans les décisions monétaires royales, a suffi à mettre la proposition de Bodin, qui avait indéniablement ses faiblesses, sur la touche.

33. Notons qu'il s'agit là encore d'une convention, non d'une loi. Pour Bodin en effet, on ne peut qualifier un prince de souverain que s'il ne doit rien à personne à l'extérieur de son territoire, c'est-à-dire si sa souveraineté interne n'est aucunement limitée par des liens juridiques externes; or la convention est précisément un lien non juridique. La souveraineté de chacune des républiques est donc sauvegardée par la nature de cette coopération internationale. Sur ces éléments de définition, voir Goyard-Fabre (1989, pp. 94-96). 
mauvaise monnaie d'un royaume voisin ou vassal a été, dans l'histoire, prétexte à une guerre ${ }^{34}$. Voici enfin fortement matérialisé l'effet d'une falsification monétaire de la part d'un prince isolé au milieu de princes vertueux en matière monétaire : la guerre. Si un souverain n'a cure des jacqueries de son royaume, s'il se défie de son peuple par son droit souverain à manipuler ses monnaies, peut-être s'arrêtera-t-il par crainte de la puissance de ses voisins. C'est à une ébauche de coopération internationale favorable à la paix, aux échanges internationaux et à la stabilité monétaire qu'appelle de ses voeux Bodin.

\section{Conclusion}

Dans les débats de l'époque, Bodin occupe une place singulière. Sa controverse avec Malestroit lui donne une certaine envergure. C'est un homme cultivé et érudit qui publie des ouvrages qui ont de l'écho et qui sait parler. Il ne parvient cependant jamais à entrer dans le cercle des conseillers du prince. Bien que, un temps, il ait l'oreille de Henri III, il perd ses faveurs lors des Etats généraux de 1576 du fait de ses prises de position à contre-courant sur leur organisation, sur les questions religieuses et sur les questions financières. Eloigné de la Cour des monnaies où s'élabore une analyse fine de la situation monétaire qui se dégrade au fil des années, il propose un système qui vise moins à régler les problèmes du moment qu'à envisager le long terme.

L'approche de Bodin est empreinte d'une grande ambiguïté quant au point de vue adopté. On peut le comprendre en distinguant deux composantes des pièces (le métal et la marque du souverain) et deux temps des pièces (la frappe et la circulation). Un point de vue strictement déterminé par sa théorie de la souveraineté devrait conduire Bodin à mettre en avant les problèmes liés à la frappe et à la marque du souverain. Ce n'est pas ce qu'il fait. Pour résoudre le problème de la fausse monnaie, profondément lié à la question de la souveraineté, il adopte au contraire un point de vue économique dans lequel ce qui importe est le métal et la circulation des espèces. Ce point de vue économique n'est pas autonomisé de ses réflexions politiques et juridiques mais il leur est au contraire subordonné.

Ainsi, le problème monétaire qui préoccupe le plus Bodin n'est pas cet afflux d'or et d'argent qu'il met bien en évidence, car il ne lui donne pas de solution : c'est la fausse monnaie, entendue comme monnaie dégradée, altérée ou contrefaite. Il n’est, dans cette problématique, guère original ; il l'est plus dans sa construction théorique du problème et dans les solutions qu'il propose.

34. Que cette mauvaise ou fausse monnaie soit avérée ou purement fantasmée : on touche là un élément essentiel de la fausse monnaie, qui est le point de vue que l'on prend pour la considérer. 
C'est contre la fausse monnaie qu'il oriente toute son analyse. L'éradiquer passe par l'élimination non seulement de la contrefaçon des particuliers, mais aussi de la dégradation des particuliers et des officiers des monnaies et enfin de l'altération du fait des princes. En subordonnant à l'élimination de ces altérations celle de la contrefaçon et de la dégradation, Bodin est conduit à soumettre la frappe au domaine de la loi et la modification des caractéristiques métalliques des monnaies et de leur cours légal au domaine du contrat. Le problème est ensuite de contraindre le souverain à respecter ses contrats. Non seulement altérer les monnaies apparait comme une rupture de la promesse du souverain, alors que Dieu même est tenu des siennes, mais en plus il met en danger la foi publique qui est un des fondements de la république. La réforme radicale du système de monnaies ${ }^{35}$ apparaît comme un élément important de sa construction, mais qui ne parvient pas à contraindre logiquement le prince à la vertu.

La solution est en définitive de forcer cette vertu en étendant au niveau international ce système de monnaies, de façon à ce que chaque prince se sente lié aux autres princes. Il s'agit là d'un apport extrêmement intéressant du point de vue de la pensée politique et monétaire. Une fois le souverain empêché par ce moyen de falsifier des monnaies au contenu immuablement pur, les officiers des monnaies et les particuliers n'auront plus aucune possibilité de contrefaire les monnaies. La fausse monnaie, dans le sens large que lui donne Bodin, sera éradiquée.

35. Nous employons ce terme plutôt que celui de système monétaire, car l'unité de compte est absente de la construction de Bodin. 


\section{Tableaux}

Tableau 1 - Typologie des fausses monnaies

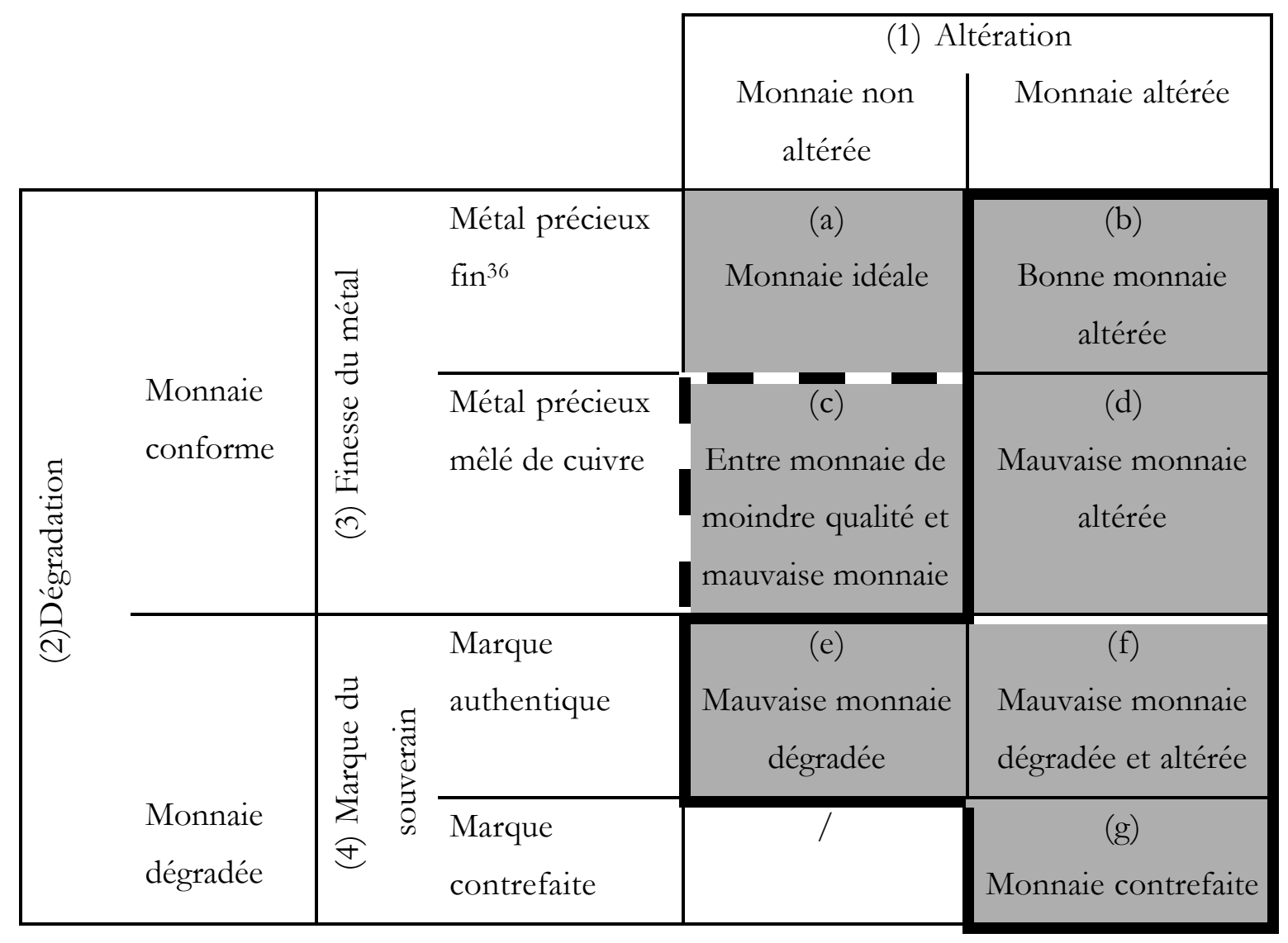

Note: ce tableau croise quatre critères: (1) le degré d'altération des pièces entendu comme l'écart entre le cours légal et le contenu métallique; (2) le degré de dégradation des pièces entendu comme l'écart entre le contenu métallique réel et le contenu métallique annoncé par ordonnance ; (3) la finesse du métal ; (4) l'authenticité de la marque du souverain. Cinq cas apparaissent comme des situations de fausse monnaie (encadrés par un trait gras : b, d, e, f, g), le cas (a) correspond à ce que recherche Bodin et le cas (c) apparaît intermédiaire, dépendant de l'importance du cuivre dans l'alliage métallique des pièces.

36. Bodin entend par là l'or fin à 23 carats et l'argent fin à 11 deniers 12 grains. 
Tableau 2 - Dimension juridique des formes de fausse monnaie

\begin{tabular}{|l|l|l|}
\hline $\begin{array}{l}\text { Type de fausse } \\
\text { monnaie }\end{array}$ & Origine & Catégorie adéquate \\
\hline Monnaie altérée & Le souverain & $\begin{array}{l}\text { Rupture de contrat et danger pour la foi } \\
\text { publique }\end{array}$ \\
\hline Monnaie dégradée & $\begin{array}{l}\text { Les officiers des } \\
\text { monnaies ou les sujets }\end{array}$ & Transgression de la loi (crime) \\
\hline Monnaie contrefaite & Les sujets & $\begin{array}{l}\text { Transgression de la loi (crime de lèse- } \\
\text { majesté) }\end{array}$ \\
\hline
\end{tabular}

\section{Bibliographie}

Arestis Philip et Howells Peter (2002), "The 1520-1640 'Great Inflation' : an Early Case of Controversy on the Nature of Money ", Journal of Post-Keynesian Economics, vol. 24, n², pp. 181-203.

Baudrillart Henri (1853), Jean Bodin et son temps. Tableau de la théorie politique et des idées économiques au XVTe siècle, Paris, Guillaumin.

Bazinghen Abot de (1764), Traité des monnoies, et de la juridiction de la Cour des monnoies, en forme de dictionnaire, 2 volumes, Paris, Guillyn.

Blanc Jérôme (1994), "Les citoyens face à la complexité monétaire : le cas de la France sous l'Ancien Régime », De Pecunia, vol. VI, n³, décembre, pp. 81-111.

Blanc Jérôme (2005), « Beyond quantitativism: a reappraisal of Jean Bodin's monetary ideas ", in Giacomin A. et Marcuzzo M. C., eds, Money and Markets. A Doctrinal Approach, London, Routledge, à paraître.

Bodin Jean (1566), La méthode de l'bistoire, Paris, Les belles lettres, 1941.

Bodin Jean (1568), « La response de Maistre Jean Bodin advocat en la Cour au Paradoxe de Monsieur de Malestroit, touchant l'encherissement de toutes choses, et les moyens d'y remedier », in Le Branchu Jean-Yves, éd., Ecrits notables sur la monnaie (XVIe siècle). De Copernic à Davanzati, volume 1, Paris, Félix Alcan, 1934, pp. 69-177.

Bodin Jean (1577), Recueil de tout ce qui s'est négocié en la compagnie du tiers Estat de France, en l'assemblée générale des trois Estats, assignez par le Roy en la ville de Bloy, au XV Novembre 1576, s.l., s.e., 119 p. 
Bodin Jean (1578), « Discours de Jean Bodin, sur le rehaussement et diminution tant d'or que d'argent, et le moyen d'y remédier, aux paradoxes du Sieur de Malestroit », in Bodin Jean, Les six livres de la République, volume 6, Paris, Fayard, 1986, pp. 415-503.

Bodin Jean (1568-1578), Response to the Paradoxes of Malestroit, Bristol, Thoemmes, 1997.

Bodin Jean (1580), Exposé du droit universel, Paris, PUF, 1985.

Bodin Jean (1583),Lessix livres de La République, Abrégé, Paris, Le Livre de Poche (Classiques de la Philosophie), 1993.

Bodin Jean (1593), Les six livres de La République, 6 volumes, Paris, Fayard, 1986.

Bodin de Saint-Laurent Jean de (1907), Les idées monétaires et commerciales de Jean Bodin, Bordeaux, thèse.

Budruss Eckhart (1987), «Erudition classique et théorie quantitative de la monnaie dans la « Réponse à Malestroit » de Jean Bodin », Journal des savants, janvier, pp. 89-125.

Franklin Julian H. (1993), Jean Bodin et la naissance de la théorie absolutiste, 1973, Paris, Presses universitaires de France (Essais).

Gonnard René (1935), Histoire des doctrines monétaires dans ses rapports avec l'histoire des monnaies, volume 1 : «De l'antiquité au XVIIe siècle », Paris, Sirey.

Goyard-Fabre Simone (1989), Jean Bodin et le droit de la république, Paris, PUF.

Guggenheim Thomas (1978), Les théories monétaires préclassiques, Genève, Droz.

Harsin Paul (1928), Les doctrines monétaires et financières en France du XVIe au XVIIIe siècle, Paris, Félix Alcan.

Hauser Henri (1932), La vie chère au XVIe siècle. La Response de Jean Bodin à M. de Malestroit, 1568, Paris, Armand Colin.

Le Branchu Jean-Yves (1934), Ecrits notables sur la monnaie (XVIe siècle) de Copernic à Davanzati, 2 volumes, Paris, Félix Alcan.

Mairet Gérard (1993), "Les six livres de la république et la fondation moderne de l'Etat profane », in Bodin Jean, Les six livres de la république, 1583, abrégé, Paris, Le livre de poche, pp. $5-38$.

Malestroit Jean Cherruyer de (1566), «Les paradoxes du seigneur de Malestroict, Conseiller du Roi et maistre ordinaire de ses comptes, sur le faict des monnoyes présentez a sa majesté, au mois de mars, MDLXVI », in Le Branchu Jean-Yves éd., Ecrits notables sur la monnaie (XVTe siècle). De Copernic à Davanzati, volume 1, Paris, Félix Alcan, pp. 49-68.

Nancey Paul (1942), Jean Bodin (1530-1596), économiste, Bordeaux, Caster.

O’Brien Dennis (2000), « Bodin's Analysis of Inflation », History of Political Economy, vol. $32, n^{\circ} 2$, pp. 267-292. 
Parsons Jotham (2003), « Governing Sixteenth-Century France : The monetary Reforms of $1577 »$, French Historical Studies, vol. 26, n²1, winter, pp. 1-30.

Sargent Thomas J. et Velde François R. (2003), The Big Problem of Small Change, Princeton, Princeton University Press.

Schumpeter Joseph Aloys (1954), Histoire de l'analyse économique, Tome 1 : "L'âge des fondateurs (Des origines à 1790) », Paris, Gallimard.

Université d'Angers et Centre de recherches en littérature et linguistique de l'Anjou et des Bocages, Jean Bodin. Actes du colloque interdisciplinaire d'Angers (24-27 mai 1984), 2 tomes, Angers, Presses de l'Université d'Angers. 